\title{
Modelling the Diclofenac and Indomethacin Recovery by Molecularly Imprinted Polymer
}

\author{
Nurlin Abu Samah, ${ }^{1,2 *}$ María-Jesús Sánchez-Martín, ${ }^{1}$ Manuel Valiente ${ }^{1}$ and \\ Montserrat López-Mesas ${ }^{1}$
}

\begin{abstract}
${ }^{1}$ Centre Grup de Tècniques de Separació en Química (GTS), Unitat de Química Analítica, Departament de Química, Universitat Autònoma de Barcelona, 08193 Bellaterra, Spain

${ }^{2}$ Faculty of Industrial Sciences and Technology, Universiti Malaysia Pahang, 26300 Pahang, Malaysia

*Corresponding author: nurlin@ump.edu.my
\end{abstract}

Published online: 25 February 2018

To cite this article: Abu Samah, N. et al. (2018). Modelling the diclofenac and indomethacin recovery by molecularly imprinted polymer. J. Phys. Sci., 29(Supp. 1), 67-74, https://doi.org/10.21315/jps2018.29.s1.9

To link to this article: https://doi.org/10.21315/jps2018.29.s1.9

\begin{abstract}
Pharmaceutical compounds in surface waters are an emerging environmental concern due to their biological activity, which consequently present a new challenge for drinking water and wastewater treatment systems. In this work, diclofenac (DCF) and indomethacin (IDM) have been used as target molecules or templates in order to be removed by means of molecularly imprinted polymers (MIP). MIP-DCF and MIPIDM were synthesised and tested using two mathematical models, which are Lagergren and Thomas models. In addition, functional groups of the active functional sites and the target molecules have also been studied by using Fourier transform infrared spectroscopy. Both MIPs followed the Lagergren pseudo first order kinetic model. Comparing the original MIP-DCF and MIP-DCF after loading with DCF, differences in their functional groups were found. In conclusion, MIP-DCF and MIP-IDM work as good sorbents to recover these emerging pharmaceuticals persistent pollutants from water.
\end{abstract}

Keywords: Molecularly imprinted polymer, MIP, diclofenac, indomethacin, diclofenac recovery

\section{INTRODUCTION}

A few decades ago, emerging persistent pharmaceutical pollutants (EPPPs) have been introduced as one type of recalcitrant pollutant sources in water. ${ }^{1}$ Recent studies have demonstrated that, despite the relatively low concentrations of 
pharmaceuticals in the environment (typically in sub-parts-per-billion levels), pharmaceuticals are of great concern due to their potential long-term adverse effects on humans and wildlife. ${ }^{2}$

Nowadays, there are many methods used for the recovery of pharmaceuticals, such as photocatalysis and sorbents, but they present some drawbacks, e.g., after photocatalysis the by-product generated may be more harmful than the parent compounds. ${ }^{3}$ There are many kinds of sorbents that have been studied, but not all the sorbents have the potential to recover the target molecules. In this work, molecularly imprinted polymers (MIPs) as a separation method is proposed and characterised. The polymeric matrices obtained using the imprinting technology are robust molecular recognition elements able to mimic natural recognition entities. ${ }^{4}$ MIPs are also good sorbents because of their porosity and their robustness properties. In this study, the removal of diclofenac (DCF) and indomethacin (IDM) have been carried out using MIPs, synthetised via bulk polymerisation with allylthiourea (AT) as the functional monomer and using DCF or IDM as template (MIP-DCF or MIP-IDM, respectively). DCF and IDM were detected using spectrophotometry.

To study the properties of the sorbents, MIPs have been tested in batch mode and continuous flow mode. In the batch mode, Lagergren model was used to describe the adsorption properties. However, in the continuous flow mode, Thomas model was used for the breakthrough study. On the other side, functional groups can be used as a fingerprint for certain MIPs to show if the active functional sites work. However, there are a few articles reporting on this parameter which give important information about MIP development. ATR-FTIR has been used to observe the different types of MIPs and to study their functional sites.

\section{EXPERIMENTAL}

\subsection{Materials}

Indomethacin (IDM) was purchased from Sigma-Aldrich, Spain, with 98\%-99\% purity (TLC grade). Diclofenac-Na was from Cayman Chemical, United States with purity of more than $99 \%$. Ethanol with $96 \%$ purity was acquired from Scharlau Chemical, Spain. Acetic acid was from J. T. Baker, United States with 99.9\% of purity, respectively, and HPLC grade. Acetonitrile (99\% of purity HPLC grade) from VWR. Grace Alltech "Extract Clean" empty reservoirs with silica frits, $1.5 \mathrm{ml}$ from Fisher Scientific, United States. 


\subsection{Synthesis of MIP-DCF and MIP-IDM}

Synthesis of MIP-IDM and MIP-DCF were performed following the procedure of Kugimiya et al. with some modifications. ${ }^{5}$

\subsection{Recovery Study}

An amount of $10 \mathrm{mg}$ MIP-IDM was weighed and placed into a $1.5 \mathrm{ml}$ cartridge. Then, $1 \mathrm{ml}$ of ethanol:water $(75 \% \mathrm{v} / \mathrm{v})$ was passed through it twice to activate the MIP-IDM. Next, $1 \mathrm{ml}$ of water was added for 5 times to rinse out the ethanol residue. Afterwards, acetonitrile:water $(5 \% \mathrm{v} / \mathrm{v})$ was added twice for conditioning the MIP-IDM. Later, $1 \mathrm{ml}$ of $15 \mu \mathrm{g} \mathrm{ml}^{-1}$ of IDM was added twice. The eluted solution was collected and analysed using a UV-vis spectrophotometer at $260 \mathrm{~nm}$. After that, $1 \mathrm{ml}$ of ethanol:water $(75 \% \mathrm{v} / \mathrm{v})$ was loaded twice into the cartridge and the collected solution was also analysed by UV spectrophotometry. The procedure was done in triplicate. The percentage of recovery was calculated using Equation 1, where $\mathrm{C}_{\mathrm{i}}$ and $\mathrm{C}_{\mathrm{f}}$ represent the analyte concentration for initial and final respectively:

$$
\% \text { Recovery }=\frac{\mathrm{C}_{\mathrm{f}}-\mathrm{C}_{\mathrm{i}}}{\mathrm{C}_{\mathrm{f}}} \times 100
$$

Moreover, the recovery procedure mentioned above was done regenerating the same MIP and reusing it until 10 times (consecutive extractions) in order to evaluate the reuse of the MIP. The same procedure was performed by using MIPDCF as a sorbent and measuring the eluted solution at $280 \mathrm{~nm}$ for DCF analysis.

\subsection{Kinetic Study}

An amount of $10 \mathrm{mg}$ of MIP-DCF was weighed and placed in $5 \mathrm{ml}$ tubes. Then, $2 \mathrm{ml}$ of $5 \mathrm{mg} \mathrm{l}^{-1}$ DCF solution were poured into the tubes and covered using aluminium foil. ${ }^{6}$ Solutions were agitated for the required time up to $120 \mathrm{~min}$ and then filtered using a syringe filter $(0.22 \mu \mathrm{m})$. The procedure was performed in triplicate and repeated for MIP-IDM using IDM solution. The calculated capacity (mg of compound/g of MIP) versus time was plotted following the Lagergren model.

\subsection{Breakthrough Experiment - Continuous Flow Mode}

Due to the immiscibility properties of DCF in water, total sorption capacity has been carried out in acetonitrile:water $(5 \% \mathrm{v} / \mathrm{v})$. An amount of $10 \mathrm{mg}$ of MIP-DCF was accurately weighed and placed in a $1.5 \mathrm{ml}$ cartridge. Next, $15 \mu \mathrm{g} \mathrm{ml}^{-1}$ of 
DCF was prepared in acetonitrile:water $(5 \% \mathrm{v} / \mathrm{v})$ and the solution was loaded into the cartridge using a peristaltic pump at $1.67 \mathrm{ml} \mathrm{min}^{-1}$. The eluted solution was collected in fractions of $5 \mathrm{ml}$ until $50 \mathrm{ml}$, then, in fractions of $50 \mathrm{ml}$ until the absorbance measurements of the eluted solution reached a plateau. All the collected fractions were analysed by UV spectrophotometry. The procedure mentioned above was done in duplicate. The same methodology was repeated in the absence of MIP, in order to measure any other contribution to the elimination of the target compounds, i.e., sorption in cartridge or frits. Similar procedure was performed for IDM removal by MIP-IDM.

\subsection{Attenuated Total Reflectance Fourier Transform Infrared Analysis}

ATR-FTIR has been used in this study to determine the functional groups in the original MIP-DCF and after loading with $2 \mathrm{ml}$ of DCF solution $\left(5 \mu \mathrm{g} \mathrm{ml}^{-1}\right)$ via molecularly imprinted solid phase extraction (MISPE).

\section{RESULTS AND DISCUSSION}

\subsection{Recovery Study and Lagergren Model}

The ability to sorb-desorb for the synthetised MIPs were not significantly decreased after 10 cycles reaching almost $100 \%$ of recovery (Figure 1). The average percentage of recovery for the 10 cycles of IDM and DCF using MIP-IDM and MIP-DCF were $98 \pm 3 \%$ and $97 \pm 2 \%$ respectively.

From the kinetics, $100 \%$ removal was obtained in $3 \mathrm{~min}$ in batch mode. It is well accepted that two-parameter adsorption kinetic equations (Lagergren firstorder and second-order) are useful tools to describe the adsorption properties of a sorbent. ${ }^{7}$ The Lagergren pseudo first-order equation can be linearly expressed as Equation 2, where $\mathrm{Q}_{\mathrm{e}}$ and $\mathrm{Q}_{\mathrm{t}}$ are the adsorption capacities (mg of compound/g of sobent) of MIP at equilibrium and at time $t$, respectively; $\mathrm{k}_{1}$ is the first order rate constant. Figure 2 shows that both MIPs fitted to the Lagergren pseudo first order model. For further experimental procedure, MIP-DCF was used as a sorbent.

$$
\operatorname{Ln}\left(\mathrm{Q}_{\mathrm{e}}-\mathrm{Q}_{\mathrm{t}}\right)=\mathrm{LnQ}_{\mathrm{e}}-\mathrm{k}_{1} \mathrm{t}
$$

\subsection{Breakthrough Study}

Breakthrough in column can be identified as the amount of influent solution passing through the bed before a maximum effluent concentration is reached. The ratio $\mathrm{C}_{\mathrm{t}}$ (effluent concentration) $/ \mathrm{C}_{\mathrm{o}}$ (influent concentration) is plotted against 


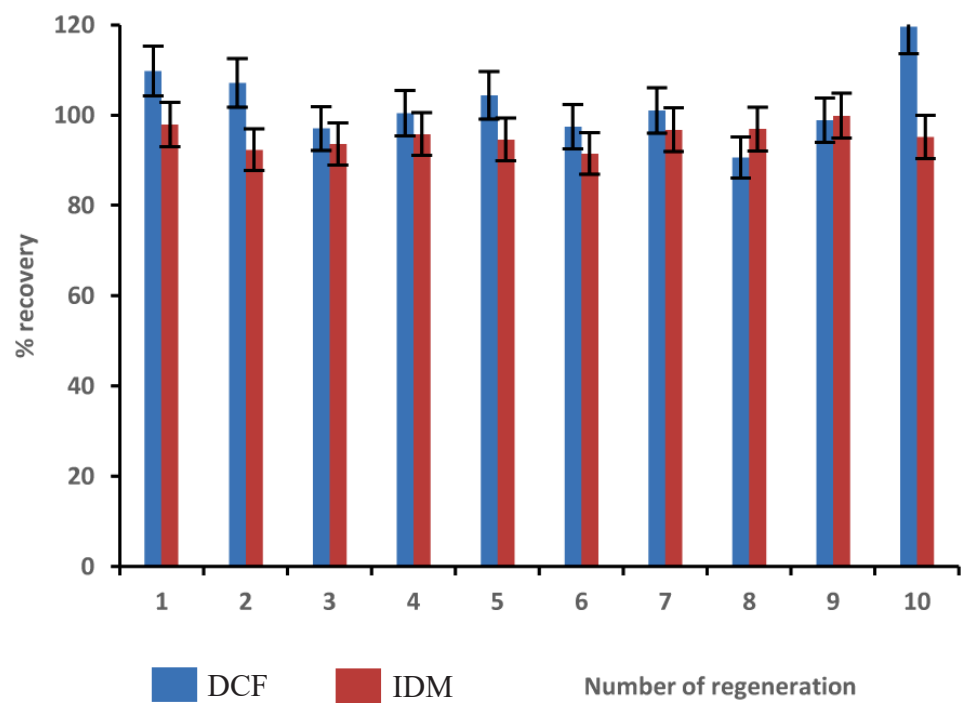

Figure 1: Percentage recovery for 10 regenerations of IDM and DCF using MIP-IDM and MIP-DCF as the sorbent respectively, via pre-packed cartridge with initial concentration of $15 \mu \mathrm{g} \mathrm{ml}^{-1}$ IDM and DCF, MIP mass: $10 \mathrm{mg}$.

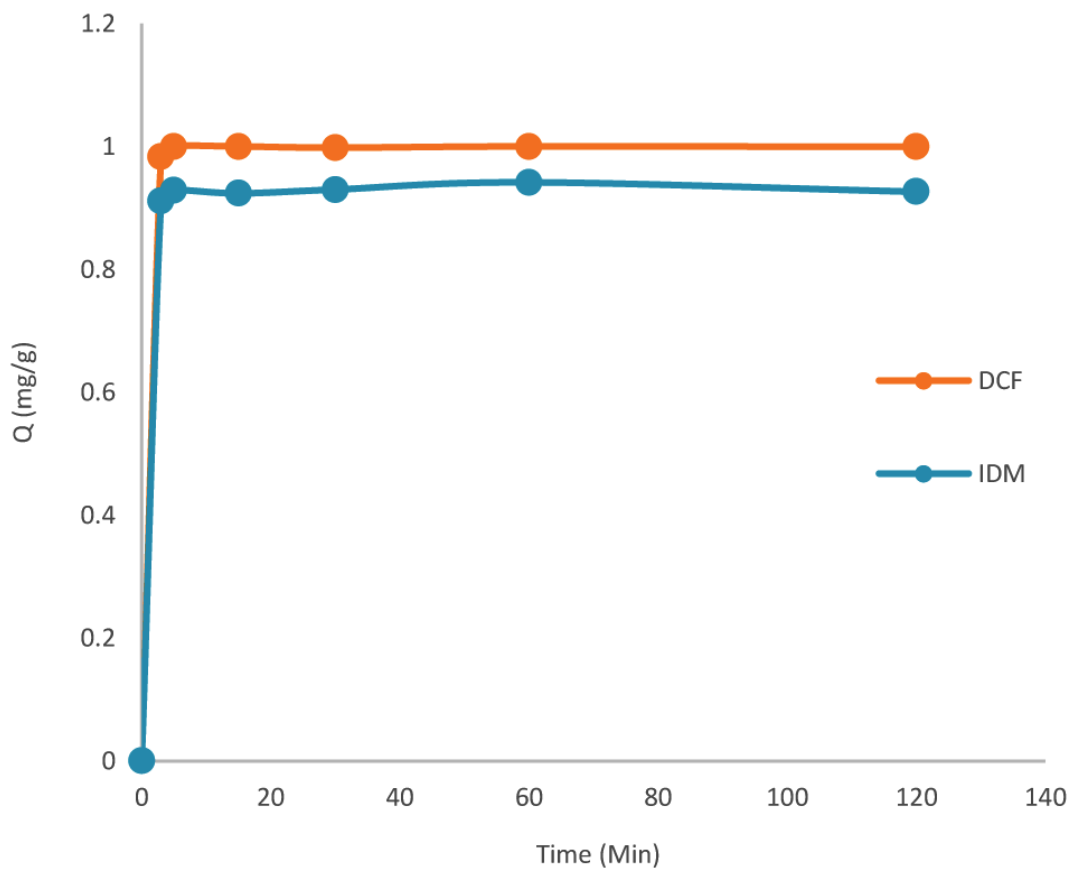

Figure 2: Adsorption capacities (Q) of MIP-IDM and MIP-DCF in front of time following Lagergren pseudo first order model plot. 
the time of the analyte solution loading as seen in Figure 3. As seen in the kinetic study, within 3 min the sorption uptake was higher than $80 \%$, thus resulting in relatively fast attainment of breakthrough. Breakthrough and exhaustion are defined as normal when the ratios of effluent-to-influent concentration are between $10 \%$ and $60 \%$, respectively. ${ }^{8}$ In this work, the breakthrough point was reached at $6 \mathrm{~min}$ and the exhaustion point at $270 \mathrm{~min}$ for MIP-DCF and $24 \mathrm{~min}$ and $210 \mathrm{~min}$ for MIP-IDM respectively. From Figure 3, it is seen that the ratio is about $60 \%$ and $80 \%$ for MIP-DCF and MIP-IDM respectively. The methodology of adsorption in fixed bed cartridge by the breakthrough curves method proved to be a good option to study the separation of the pharmaceutical compounds in water.

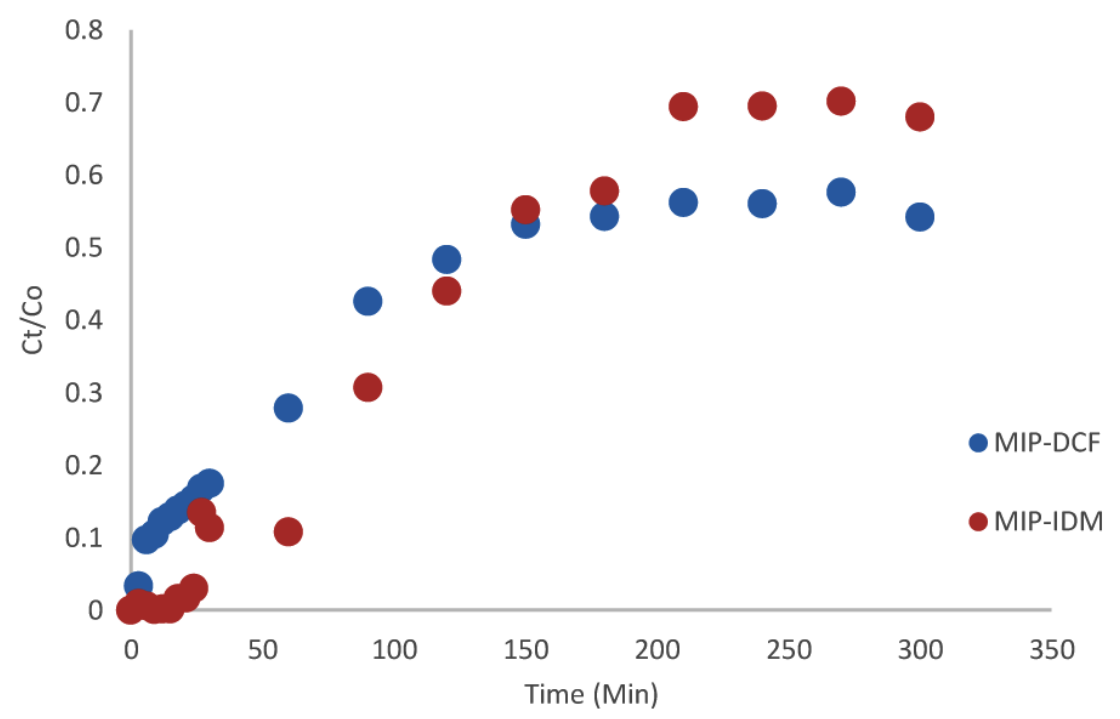

Figure 3: Breakthrough curves from packed cartridges using MIP-DCF as sorbent for DCF removal and MIP-IDM as sorbent for IDM removal, using Thomas model versus time.

\subsection{FTIR Analysis}

The MIPs, original and after DCF loading, have been differentiated via ATR-FTIR and the spectra obtained could be used as a fingerprint in order to distinguish them as seen in Figures 4(a) and 4(b). N-H stretch functional group at $3000 \mathrm{~nm}^{-1}$ and C-N stretch bond at $1180 \mathrm{~nm}^{-1}$ found in original MIP (Figure 4(a)); OH broad peak at $3300 \mathrm{~nm}^{-1}$ found in MIP loaded with DCF (Figure 4(b)) due to -OH functional group from DCF bonded to MIP-DCF. Probably there is also N-H functional group that might contribute in this peak; $\mathrm{C}=\mathrm{O}$ bond at $1750 \mathrm{~nm}^{-1}$ found in all MIPs; and $\mathrm{C}=\mathrm{C}$ bond at $1650 \mathrm{~nm}^{-1}$ found in MIP loaded with DCF. 
(a)
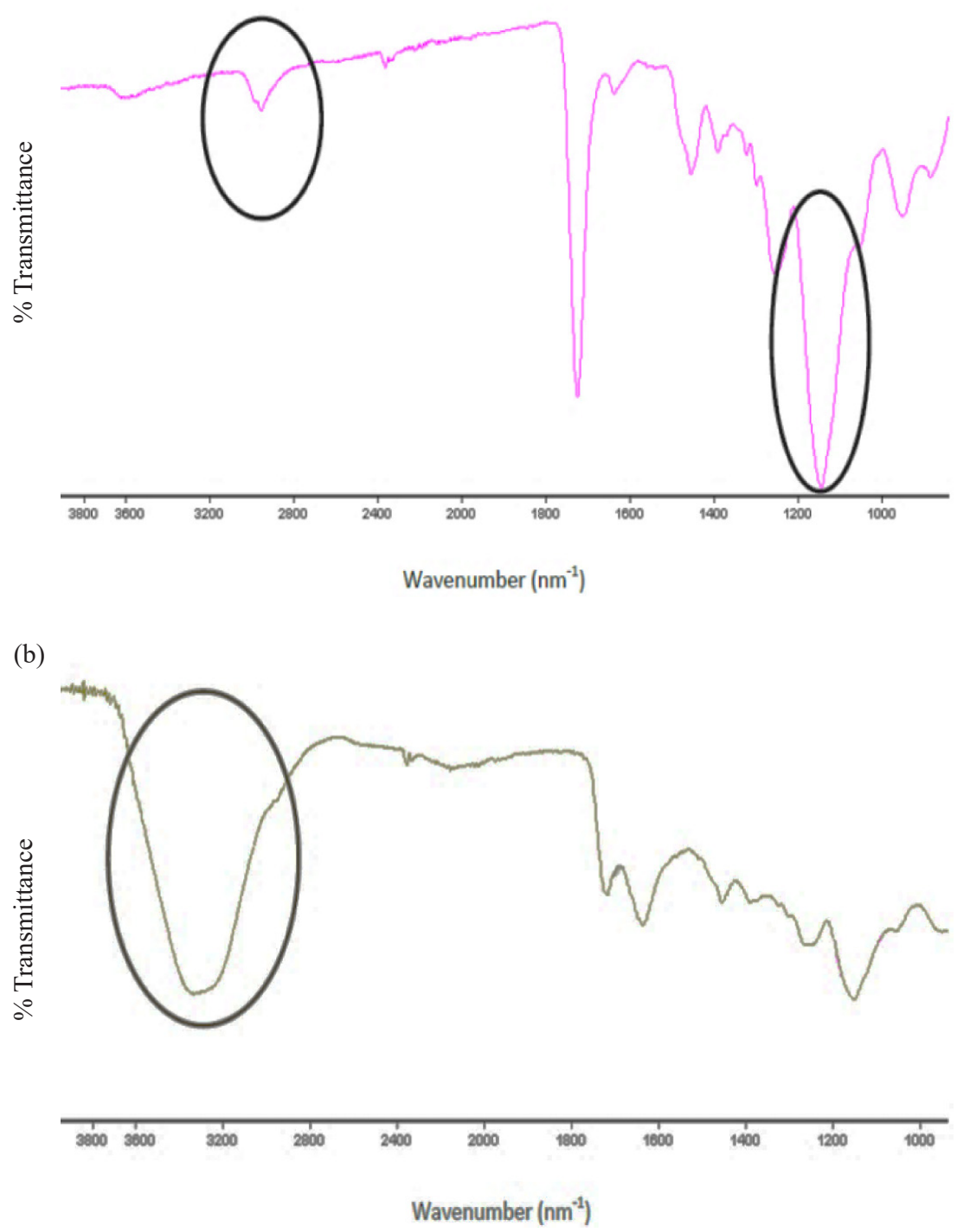

Figure 4: Infrared spectrum of (a) original MIP-DCF and (b) MIP-DCF loaded with DCF.

\section{CONCLUSION}

In conclusion, MIP-DCF and MIP-IDM have shown to be highly effective for the removal of the pharmaceutical compounds DCF and IDM, respectively. MIP is a promising analytical method for the removal of pharmaceuticals from water. 


\section{ACKNOWLEDGEMENTS}

The authors thank the Spanish Project CTM 2015-65414-C2-1-R, Skim Latihan Akademik IPTA (SLAI) by Ministry of Higher Education, Malaysia and Universiti Malaysia Pahang, Malaysia for their financial support.

\section{REFERENCES}

1. Iglesias, A. et al. (2014). Detection of veterinary drug residues in surface waters collected nearby farming areas in Galicia, North of Spain. Environ. Sci. Pollut. Res., 21, 2367-2377, https://doi.org/10.1007/s11356-013-2142-7.

2. Celiz, M. D., Tso, J. \& Aga, D. S. (2009). Pharmaceutical metabolites in the environment: analytical challenges and ecological risks. Environ. Toxicol. Chem., 28, 2473-2484, https://doi.org/10.1897/09-173.1.

3. Basha, S. et al. (2010). Studies on the adsorption and kinetics of photodegradation of pharmaceutical compound, indomethacin using novel photocatalytic adsorbents (IPCAs). Ind. Eng. Chem. Res., 49, 11302-11309, https://doi.org/10.1021/ie101304a.

4. Vasapollo, G. et al. (2011). Molecularly imprinted polymers: Present and future prospective. Int. J. Mol. Sci., 12, 5908-5945, https://doi.org/10.3390/ ijms 12095908.

5. Kugimiya, A. \& Takei, H. (2006). Preparation of molecularly imprinted polymers with thiourea group for phosphate. Anal. Chim. Acta, 564, 179183, https://doi.org/10.1016/j.aca.2006.01.099.

6. Yang, T. et al. (2008). Development of a selective molecularly imprinted polymer-based solid-phase extraction for indomethacin from water samples. Anal. Bioanal. Chem., 391, 2905-2914, https://doi.org/10.1007/s00216008-2218-2.

7. Ho, Y. S. \& McKay, G. A. (1998). Comparison of chemisorption kinetic models applied to pollutant removal on various sorbents. Process Saf. Environ. Prot., 76, 332-340, https://doi.org/10.1205/095758298529696.

8. Sadaf, S. \& Bhatti, H. N. (2014). Batch and fixed bed column studies for the removal of Indosol Yellow BG dye by peanut husk. J. Taiwan Inst. Chem. Eng., 45, 541-553, https://doi.org/10.1016/j.jtice.2013.05.004. 